\title{
A discrete Gauss-Bonnet type theorem
}

\section{Oliver Knill}

Oliver Knill hat an der ETH Zürich in Mathematik promoviert. Seit zehn Jahren wirkt er an der Harvard Universität im Preceptor Team, das dort für das Calculus Programm verantwortlich ist.

In memory of Ernst Specker.

\section{Introduction}

For a domain $G$ in the plane with smooth boundary $C$, the Gauss-Bonnet formula or " $U m$ laufsatz" $\int_{C} \kappa(s) d s=2 \pi \chi(G)$ relates the curvature $\kappa(s)$ of the boundary curve $C$ with the Euler characteristic $\chi$ of the region $G$. For a simply connected smooth region $G$ for which the boundary is a simple closed curve, the total boundary curvature is $2 \pi$. Hopf's "Umlaufsatz" relates a differential geometric quantity, the boundary curvature, with a topological invariant, the Euler characteristic. In differential geometry, curvature needs a differentiable structure, while Euler characteristic does not. It is the transcending property between different mathematical branches which makes Gauss-Bonnet type results interesting. The discrete version of the classical Hopf "Umlaufsatz" [4] is of combinatorial nature; curvature is an integer. The result applies to some 2-dimensional graphs which are

Es sei $G$ ein Gebiet in der Ebene mit glattem Rand $C$. Dann lässt sich die Krümmung $\kappa(p)$ für einen Punkt $p \in C$ wie folgt berechnen: Bezeichnet $\left|S_{r}(p)\right|$ die Länge des Kreisbogens, der aus Punkten in $G$ mit Abstand $r$ zu $p$ besteht, so gilt $\kappa(p)=$ $\lim _{r \rightarrow 0}\left(2\left|S_{r}(p)\right|-\left|S_{2 r}(p)\right|\right) /\left(2 r^{2}\right)$. Diese Formel motiviert es, für Graphen die Krümmung $K(p)$ durch die Formel $2\left|S_{1}(p)\right|-\left|S_{2}(p)\right|$ zu definieren, wo $\left|S_{1}(p)\right|$ und $\left|S_{2}(p)\right|$ die Kreise mit Radius 1 und 2 im Graphen sind. In der vorliegenden Arbeit untersucht der Autor diese Krümmung für Graphen $G$, die Teil eines flachen hexagonalen Gitters $X$ sind. Im klassischen Fall sagt ein Umlaufsatz von Heinz Hopf, dass die totale Randkrümmung $\int_{C} \kappa(p) /(2 \pi)$ mit der Euler-Charakteristik des Gebietes übereinstimmt. Hier wird gezeigt, dass für eine Klasse von Graphen $G$ in $X$ die Summe der Randkrümmungen genau zwölf mal die Euler-Charakteristik von $G$ ist. 
part of a flat 2-dimensional background graph $X$, where dimensionality is defined inductively. We need some assumptions on $G$ to equate the total boundary curvature with the Euler characteristic.

We use the curvature $K(p)=2\left|S_{1}(p)\right|-\left|S_{2}(p)\right|$, where $\left|S_{r}(p)\right|$ is the arc length of the sphere $S_{r}(p)$ at the point $p$. The sphere $S_{r}(p)$ is a subgraph of $G$ containing as vertices points of geodesic distance $r$ and edges consisting of pairs $\left(q, q^{\prime}\right)$ in $S_{r}(p)$ such that $q$ and $q^{\prime}$ have distance 1 . Gauss-Bonnet type results are more subtle for this curvature $K$ than for the first order curvature $K_{1}(p)=6-\left|S_{1}(p)\right|$ in the interior or $K_{1}(p)=3-\left|S_{1}(p)\right|$ at the boundary. While $\sum_{p \in G} K_{1}(p)=6 \chi(G)$ holds for any 2-dimensional graph $G$ with 1dimensional boundary, the sum $\sum_{p \in G} K(p)$ of all curvatures $K(p)=2\left|S_{1}(p)\right|-\left|S_{2}(p)\right|$ is only equal to $30 \chi(G)$, if more smoothness is present for the 2-dimensional graph $G$. In this article, we see this subtlety to appear in a simpler "Umlaufsatz" situation, where the graph is a subgraph of a fixed flat background lattice.

The main result in this paper is the formula $\sum_{p \in \partial G} K(p)=12 \chi(G)$ which holds for discrete domains $G$ and for the second order curvature $K$. To do so, we need to specify precisely what a smooth domain is. The background lattice $X$ plays the role of the 2dimensional plane. Its vertices are the set of points $\{k(1,0)+l(1, \sqrt{3}) / 2 \mid k, l \in \mathbb{Z}\}$. The edges consist of pairs for which the Euclidean distance is 1 . In the infinite graph $X$, every point $p$ has six neighbors. Together with edges formed by neighboring vertices, these points form the unit sphere $S_{1}(p)$, a subgraph of $X$. Similarly, any sphere $S_{2}(p)$ of radius 2 in this discrete plane has length $\left|S_{2}\right|=12$. The curvature $K=2\left|S_{1}\right|-\left|S_{2}\right|$ is zero at every point of the background lattice $X$.

\section{Topology of the planar triangular lattice}

A subset $G$ of the triangular lattice $X$ defines a $\operatorname{graph}(V, E)$, where $V \subset X$ is the set of vertices in $G$ and where $E$ is a subset of edges $(p, q)$ in $X$, pairs in $V$ having distance 1 within $X$. We often equate $G$ with the vertex set $V$ because $V$ defines the graph $G$ for the situations we are interested in. We start by defining a dimension for graphs. To our best knowledge, this notion has not yet have appeared, even though in the graph theory literature, several notions of dimension exist. The inductive definition of dimension is rather general and does not require the graph to be a subset of $X$.

Definition. A sphere $S_{r}(p)$ is a subgraph $G$ of $X$ whose vertices are the set of points in $G$ which have geodesic distance $r$ to $p$ normalized so that adjacent points have distance 1 within $G$. The edges of the sphere $S_{r}$ are all pairs $(p, q)$ with $p, q \in S_{r}(p)$ for which $(p, q)$ is in $E$. A disc $B_{r}(p)$ in the graph $G$ is the set of points $q$ which have distance $d(q, p) \leq r$ in $G$. A vertex $p$ of a graph $G=(V, E)$ is called 0-dimensional, if $p$ is not connected to any other vertex. A subgraph $G$ of $X$ is called 0-dimensional, if every point $p \in G$ is 0 -dimensional in $G$. Such a graph has no edges. A point $p$ of $V$ is called 1-dimensional, if $S_{1}(p)$ is 0 -dimensional, where $S_{1}(p)$ is the unit sphere of $p$ within $G$. A finite subset $G$ of $X$ is called 1-dimensional, if any of the points in $G$ is 1-dimensional. A point $p$ of $G$ is called 2-dimensional, if $S_{1}(p)$ is a 1-dimensional graph. A subset $G$ of $X$ is called 2-dimensional, if every vertex $p$ of $G$ is 2-dimensional. 


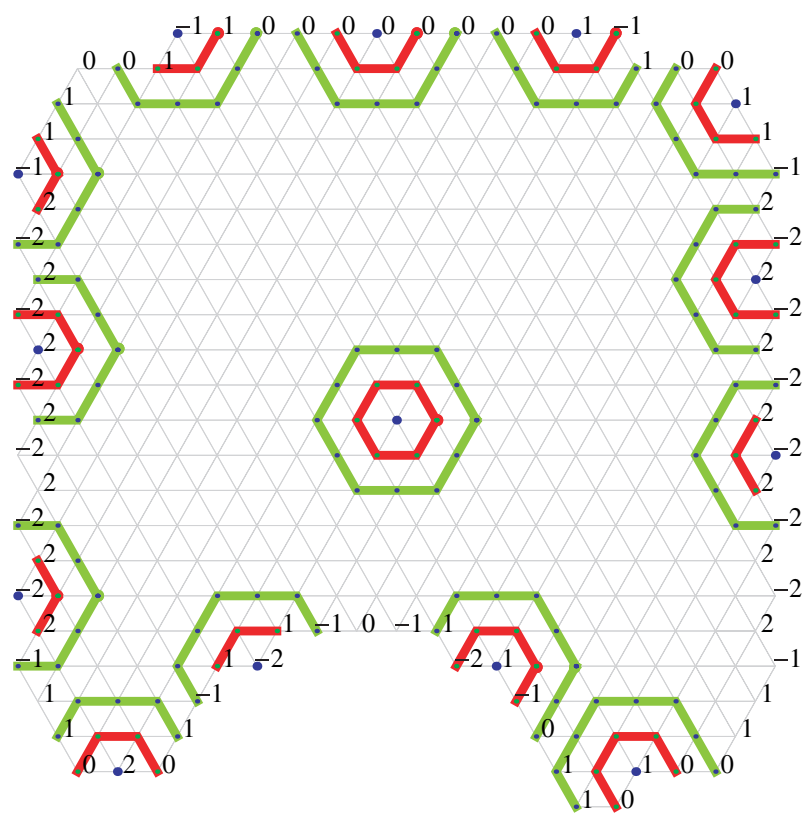

Fig. 1 The numbers near each vertex indicate the curvature of the point. At each of a few chosen points, the spheres of radius 1 and 2 in $G$ are drawn. Adding up the curvatures over the boundary gives 12. If a point has as a neighborhood a disc of radius 2 , the curvature is zero.

\section{Remarks.}

a) To illustrate this, we look at the Platonic solids. The cube and the dodecahedron are 1-dimensional. The isocahedron and octahedron are 2-dimensional. The tetrahedron is 3-dimensional. The cube and dodecahedron become 2-dimensional after a stellation of faces. The tetrahedron becomes 2-dimensional after truncating corners and then stellating the hexagonal faces.

b) Dimension could be generalized by defining inductively a fractional dimension by adding 1 to the average fractional dimensions of points in $S_{1}(p)$.

Definition. A point $p$ in $G$ is called an interior point of $G$, if the sphere $S_{1}(p)$ in the graph $G$ is the same as the sphere $S_{1}(p)$ in the background graph $X$. In other words, for an interior point, the sphere $S_{1}(p)$ is a connected 1-dimensional graph without boundary, a closed circle. A point $p$ of a 2-dimensional graph $G$ is a boundary point of $G$, if it is not an interior point in $G$ but has a neighbor in $G$ which is an interior point. For a boundary point, the sphere $S_{1}(p)$ is a union of 1-dimensional arcs. The boundary of $G$ is the set of boundary points of $G$. The interior of $G$ is the set of interior points of $G$.

\section{Remarks.}

a) The set of subsets $\{A \subset \operatorname{int}(G)\} \cup\{G\}$ defines a topology on $G$ such that the interior of $G$ is open and the boundary $\partial G$ is closed. 
b) The interior of a 2-dimensional graph $G$ is not necessarily 2-dimensional. The disc of radius 1 in $X$ for example has a single interior point so that the interior is 0 dimensional.

c) 2-dimensionality of a graph has no relation with being planar. The tetrahedral graph for example is 3-dimensional in our sense but planar. A triangularization of a torus like seen in Fig. 16 is 2-dimensional but not planar.

d) Topologically, the triangular graph $X$ is the only simply connected 2-dimensional flat graph without boundary.

Definition. A subset $G$ of $X$ is a domain, if the following five conditions are satisfied:

(i) $G$ is a 2-dimensional subgraph of $X$.

(ii) Every point of $G$ is either an interior point or a boundary point.

(iii) The set of boundary points in $G$ is a 1-dimensional graph.

(iv) If two vertices $p, q$ in $G$ have distance 1 in $X$, then $(p, q)$ is an edge in $G$.

(v) Two interior points in $G$ with a common boundary point have distance 1 or are both adjacent to a third interior point.

A domain $G$ is called a finite domain, if it is a finite graph which is a domain. $G$ is called a smooth domain, if $G$ and its complement are both domains.

The assumptions (i), (ii), (iii) are natural. Condition (iv) assures that no unnatural fissures can exist. Condition (v) assures that the connectivity topology of the domain and the connectivity topology of the interior set are the same.

\section{Remarks.}

a) We could require the interior of a domain to be 2-dimensional but prefer not to do that because the proof of Theorem 2 simplifies, if we do not make this assumption. The additional requirement would lift a difficulty on a different level. For us it will be important to look at the dimension of points with respect to the interior of $G$.

b) The conditions (i)-(v) have analogue statements in the continuous case, where they are necessary for the classical Gauss-Bonnet formula to be true: we cannot have hairs sticking out of the domain for example. The closure of the complement of a domain is a domain too and we cannot just leave out part of the boundary. Also in the continuous case, it should not happen that parts of domains are tangent to each other. We also cannot allow the boundary to be 2-dimensional, like for the Mandelbrot set.

c) For a smooth domain, we can look at the interior $H^{\prime}=\operatorname{int}\left(G^{\prime}\right)$ of the complement $G^{\prime}$ of $\operatorname{int}(G)$. Then, the boundaries satisfy $\partial G=\partial G^{\prime}$. The three sets $\operatorname{int}\left(G^{\prime}\right), \operatorname{int}(G)$, and $\partial G=\partial G^{\prime}$ partition the graph $X$.

The following lemma allows us to deal efficiently with eligible regions and eliminates many subsets which are not regions. It says that the set of interior points determines the region as well as its boundary.

Lemma 1. Let $G$ be a domain and $H=\operatorname{int}(G)$ be the set of interior points of $G$. Then $G=\bigcup_{q \in H} B_{1}(q)$, where $B_{1}(q)$ is the disc of radius 1 in $X$. Especially, the interior set $H=\operatorname{int}(G)$ determines the domain $G$ completely. 

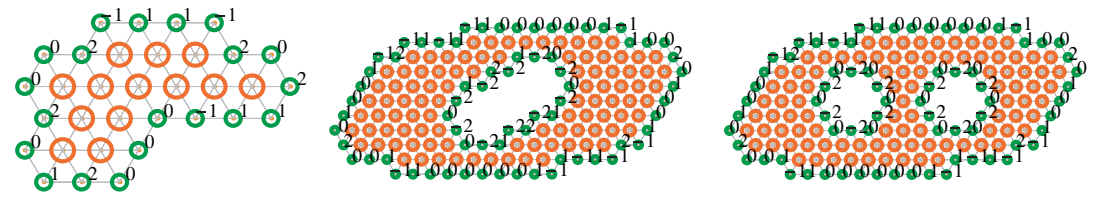

Fig. 2 Examples of domains. In each of the three pictures, the integer in the upper right corner is the total boundary curvature of the domain.

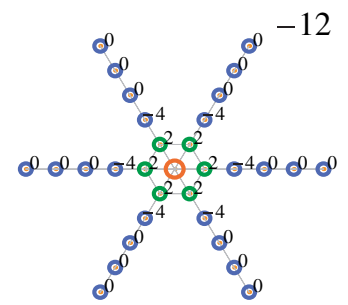

4
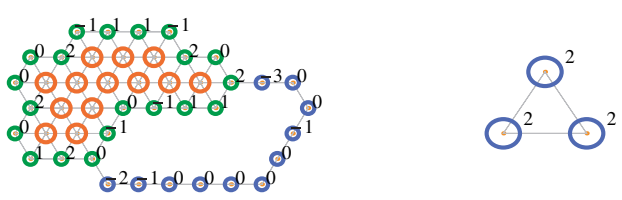

Fig. 3 Examples of graphs which are not domains. To the left, a set with 2-, 1-, and 0-dimensional points. It violates condition (i). The second example is a set with both 2- and 1-dimensional points. The third example is a 2-dimensional set with no interior points and no boundary points. It violates condition (ii).

Proof. If a point $p$ is in $G$, then it is either an interior point or a point adjacent to an interior point. Therefore, $G \subset \bigcup_{q \in H} B_{1}(q)$. On the other hand, if $p$ is in $\bigcup_{q \in H} B_{1}(q)$, then $p \in B_{1}(q)$ for some $q$. Because $q \in G$ and $S_{1}(q) \subset G$ by definition of being an interior point, we have $p \in G$.

\section{Curvature}

Definition. Let $\left|S_{r}(p)\right|$ denote the number of edges in the sphere $S_{r}(p)$. We call it the arc length of the sphere $S_{r}$.

Note that $\left|S_{1}\right|$ is not necessarily the number of vertices in $S_{1}$. Similarly, $\left|S_{2}\right|$ is the number of edges in $S_{2}$ which is not always equal to the number of vertices in $S_{2}$.

Definition. The curvature of a boundary vertex $p$ in a region $G$ is defined as

$$
K(p)=2\left|S_{1}(p)\right|-\left|S_{2}(p)\right| .
$$

The curvature of a finite domain $G$ is the sum of the curvatures over the boundary.

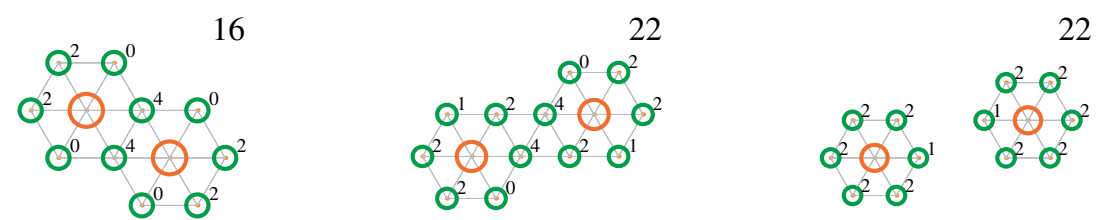

Fig. 4 Examples of graphs which are not smooth domains. The first violates (v), the second violates (iii), the third has a complement which is not a smooth domain. 


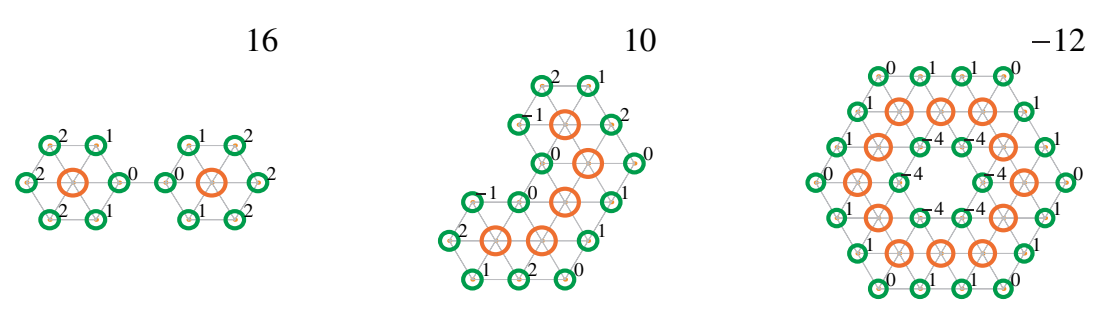

Fig. 5 More graphs which are not smooth domains. The curvature of the first was computed while assuming the nearest neighbor connection to be an edge as required by condition (iv). In all cases the complement is not a region.
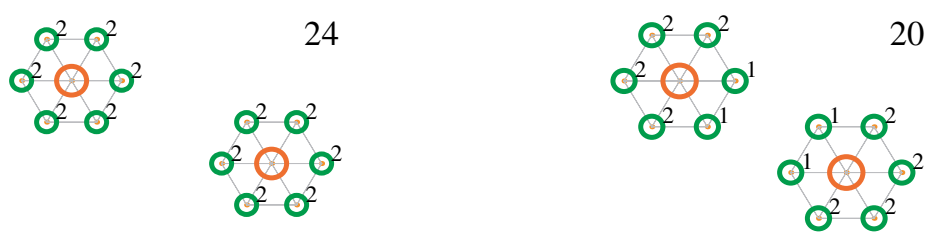

Fig. 6 The first picture is a smooth domain. It is not connected. The two parts $S_{1}, S_{2}$ of the regions are at first separated enough to get curvature 24 . In the second case, the sphere $S_{2}$ feels part of the other region $S_{1}$ and the total curvature of the boundary is not a multiple of 12 .

\section{Remarks.}

a) This definition is motivated by differential geometry where the curvature in the interior of a smooth surface is

$$
\kappa(p)=\lim _{r \rightarrow 0} \frac{2\left|S_{r}(p)\right|-\left|S_{2 r}(p)\right|}{2 \pi r^{3}}
$$

and the curvature of a smooth curve in the plane is

$$
\kappa(p)=\lim _{r \rightarrow 0} \frac{2\left|S_{r}(p)\right|-\left|S_{2 r}(p)\right|}{2 r^{2}}
$$

for a point $p$ on the boundary of a flat region $G$ and where $\left|S_{r}(p)\right|$ is the arc-length of $S_{r}(p) \cap G$.

b) Every point $q \in G$ of distance 2 in $X$ to $p \in G$ belongs to $S_{2}(p)$ whether there is a connection within $G$ from $p$ to $q$ or not.

Definition. A curve $\gamma$ in a smooth domain $G$ is a sequence of points $x_{0}, \ldots, x_{n}$ in the interior of $G$ such that $d\left(x_{i}, x_{i+1}\right)=1$ and consequently $\left(x_{i}, x_{i+1}\right)$ is an edge of $G$. A curve is a closed curve, if $x_{0}=x_{n}$. In graph theory, a curve is also called a chain. A closed curve is a non-trivial closed curve, if its length is larger than 1 . It is called a simple closed curve, if all points $x_{0}, x_{1}, \ldots, x_{n-1}$ are different and $x_{0}=x_{n}$. A domain is called simply connected, if every closed curve $\left\{x_{1}, \ldots, x_{n}\right\}$ in the interior $H$ of $G$ can be deformed to a trivial closed curve within $G$, where a deformation of a curve within $G$ is a composition of finitely many elementary deformation steps $\left\{x_{1}, \ldots, x_{n}\right\} \rightarrow\left\{y_{1}, \ldots, y_{m}\right\}$ with $\sum_{i} d\left(x_{i}, y_{j(i)}\right)=1$ and such that $x_{i}, y_{j(i)}$ are in $H$ and $d(x, y)$ is the geodesic 
distance in the graph with $d(x, y)=1$, if $(x, y)$ is an edge. As in the continuous case, simply connectedness means that any closed curve in the interior of $G$ can be deformed to a point within the interior of $G$.

\section{The curvature 12 theorem}

The main result of this paper is a discrete version of the "Umlaufsatz". It will be generalized to more general domains below.

Theorem 2 (Curvature 12 "Umlaufsatz"). The total boundary curvature of a finite, smooth, and simply connected domain $G$ is 12 .

Proof. For the proof, it suffices to look at local deformations of the region. We start with an arbitrary finite, simply connected, smooth region $G$ and find a procedure to remove interior points near the boundary while keeping it simply connected and also preserve the curvature. Removing one point affects the curvatures in a disc of radius 2 only:

Lemma 3 (Curvature is local). Let $G_{1}, G_{2}$ be two regions and $p$ be a point in both $G_{1}$ and $G_{2}$. Let $U_{1}=B_{1}(p) \subset G_{1}$ and $U_{2}=B_{2}(p) \subset G_{2}$ be the discs of radius 2 in $G_{1}$ and $G_{2}$, respectively. Define $H_{i}=G_{i} \backslash\{p\}$. If $U_{1}=U_{2}$, then

$$
\sum_{p \in H_{1}} K(p)-\sum_{p \in H_{2}} K(p)=\sum_{p \in G_{1}} K(p)-\sum_{p \in G_{2}} K(p) .
$$

The lemma tells that, if we remove a point from a region, then the total curvature-change can be read off from the curvature-changes in a disc of radius 2 . We could therefore check all possible configurations in discs of radius 2 and compare the total curvature before and after the center point is removed. We indeed did this with the help of a computer. This helped us also to identify the failure cases and to define what a domain is.

But checking all possible local deformations is not a complete proof yet. We also need to know that there is always a point which we can remove without changing the topology of $G$ or its complement. It turns out that this question is of more global nature. Take a ring shaped region for example which has a 1-dimensional interior. No point can be removed without the curvature to change. See the right picture in Fig. 12. The key is to look at the dimension of points in the interior of $G$ and distinguish points which are 1-dimensional in $\operatorname{int}(G)$ and points which are 2-dimensional in $\operatorname{int}(G)$. A 0-dimensional interior means for a simply connected region that the graph is the disc of radius 1 in $X$. By removing interior points, we want to reach this situation.

We can focus on the topology of interior points because $\operatorname{int}(G)$ defines $G$ by Lemma 1 . It is enough to check therefore what happens, if we remove interior points. Our goal is to show:

Proposition 4 (Trimming a tree). For any simply connected smooth region $G$ for which the interior set $H$ has more than one point, it is possible to remove an interior point $p$ from $H$, such that the new region defined by $H \backslash\{p\}$ remains a simply connected smooth region with one interior point less and such that the curvature does not change. 

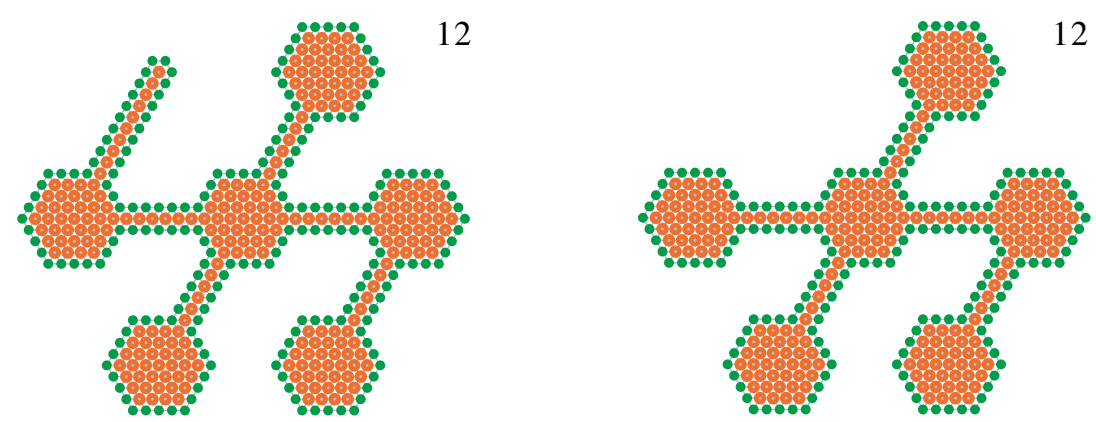

Fig. 7 The picture illustrates the process of pruning a tree. To shrink the region, we have to remove alternatively 2- or 1-dimensional interior points until only one interior point is left. The left picture shows a simply connected region where one branch can be trimmed. We get the tree to the right, where only ridges and bridges are left and all branches have been pruned. We would now have to start etching the ridges. There are four end ridges to choose from. The simple connectivity assures that at least one end ridge exists.

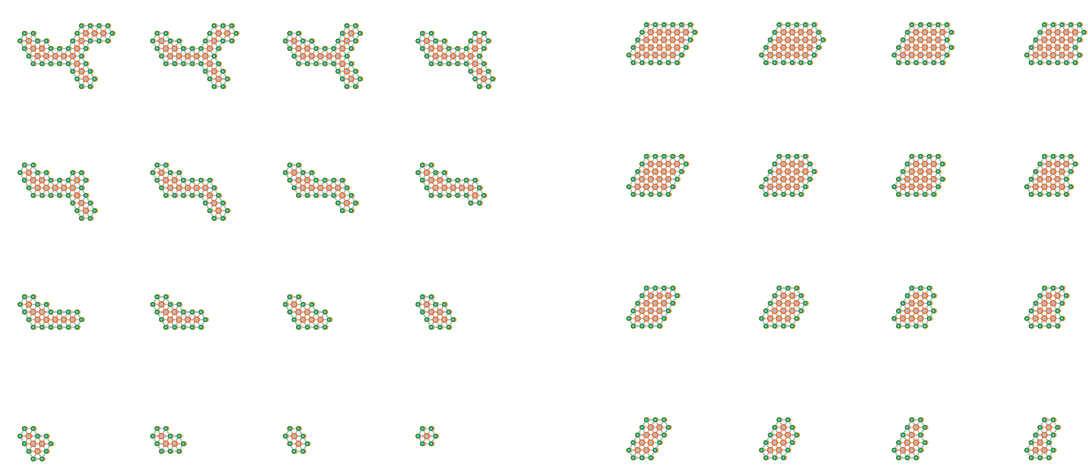

Fig. 8 The left picture shows the process of trimming of branches, the right picture shows a sequence of edging steps. Proposition 4 claims that one of the steps can be applied to a simply connected region with more than one interior point.

Theorem 2 follows from this proposition. To prove the latter, we need some more terminology:

Definition. Assume we are given a smooth and simply connected region $G$ with interior $H$. Denote by $H_{1}$ the points in $H$ which are 1-dimensional in $H$. Similarly, call $H_{2}$ the set of points in $H$ which are 2-dimensional in $H$. Connected components of $H_{1}$ are called either branches or bridges. Connected components of $\mathrm{H}_{2}$ are called ridges. A branch of $G$ is a connected component of $H_{1}$ for which at least one point has only one interior neighbor. All other connected components of $H_{1}$ are called bridges.

The set $\operatorname{int}(G)$ is the union of points which are 2-dimensional in $\operatorname{int}(G)$ and points which are 1-dimensional in $\operatorname{int}(G)$. We will use two procedures called pruning and etching to make the region smaller. The pruning procedure removes a 1-dimensional interior point at branches. The etching procedure removes a 2-dimensional interior point at ridges. 
12

$0^{1} O^{2} O^{1}$
$0^{2} 0^{12} O^{2}$
$0^{1} O^{2} O^{1}$

12

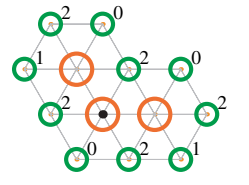

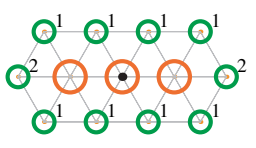

12

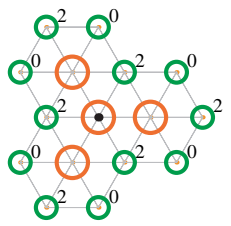

Fig. 9 The figure shows a 1-dimensional interior point and its possible neighbors. It can have either one, two, or three neighbors. There are four possibilities up to rotation. The situation of four or more neighbors makes the interior point 2-dimensional in $\operatorname{int}(G)$.

Let us start with the pruning procedure which removes interior points which are 1dimensional in $\operatorname{int}(G)$. It allows us to remove 1-dimensional branches until we can no more reduce 1-dimensional points in $\operatorname{int}(G)$. Removing 1-dimensional parts will make sure that there will be 2-dimensional ridges ready for the etching procedure. If edging is no more possible, we will reach a situation where pruning needs to be done again. Fig. 9 shows the situations which can occur locally at a point of a branch.

After reducing 1-dimensional branches, the tree still can have 1-dimensional parts: these are 2-dimensional ridges connected with 1-dimensional bridges which cannot be pruned immediately without changing the topology.

The etching procedure is invoked, if no 1-dimensional branches are left any more. The region consists now of 2-dimensional ridges connected with bridges. Our goal is to see that we can remove a 2-dimensional interior point of a ridge.

The simply connectivity implies that there is a ridge which has only one bridge connected to it. To see this, look at a new graph, which contains the 2-dimensional ridges as vertices and 1-dimensional bridges as edges. This graph contains no closed curves which are not contractible, is connected and must therefore be a tree with at least one end point. We can consequently focus our discussion to such an end-ridge for which only one 1-dimensional bridge is attached. We are able to remove a boundary point on the opposite side of that region, where no branches can be and where the boundary is smooth.

Once the etching process is over, we can again start pruning branches, or we are left with a region with only one interior point. If a region $G$ can no more be pruned and edged then $\operatorname{int}(G)$ consists of only one point and $G$ consists of only seven points and in this case, we know the total curvature is 12 .

Since pruning and etching did not change the curvature, we demonstrated that one can reduce every simply connected region to a situation with only one interior point. This completes the proof of the curvature 12 theorem. 

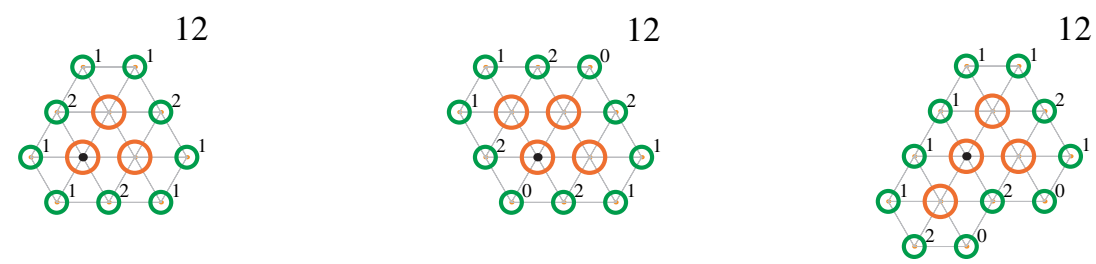

Fig. 10 A 2-dimensional interior point at the boundary can have either two or three or four interior points as neighbors. We see all the possible configurations with either two or three interior neighbors up to a rotation or a reflection.

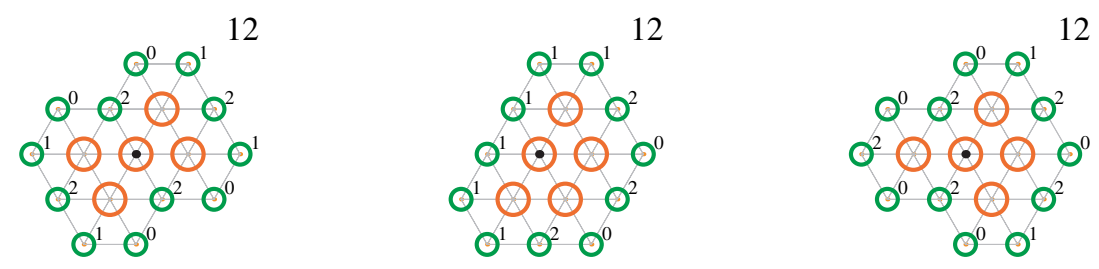

Fig. 11 Situations, where a 2-dimensional interior point at the boundary has four interior points as neighbors. In all these cases, the middle point cannot be removed while keeping the region a smooth region. If a point has five interior points as neighbors, it automatically must have six neighbors and is no more connected to a boundary point of $G$.

\section{Discrete Gauss-Bonnet theorem}

To generalize the "Umlaufsatz" to domains which are not necessarily simply connected we first define the Euler characteristic of a region using Euler's formula:

Definition. A face in a domain $G=(V, E)$ is a triangle $(p, q, r)$ of three points in $G$ for which all three points have mutual distance 1. An edge in $G$ is a pair $p, q$ of points in $G$ of distance 1. A vertex is a point in $G$. Denote by $f$ the number of faces in $G$, by $e=|E|$ the number of edges, and $v=|V|$ the number of vertices. The Euler characteristic $\chi(G)$ of the domain $G$ is defined as $\chi(G)=v-e+f$.

Remark. The Euler characteristic of $\operatorname{int}(G)$ and $G$ is the same, if $G$ is a smooth region but we do not need this fact. For a simply connected region for example the Euler characteristic is 1 .

12

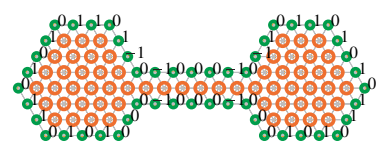

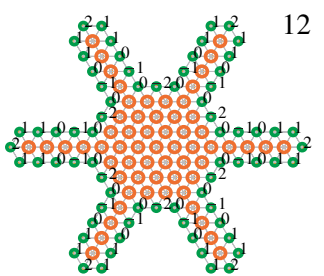

0

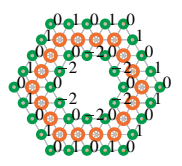

Fig. 12 The left picture shows a bridge. No interior point which is 1-dimensional in $\operatorname{int}(G)$ can be removed. The middle picture shows a situation where no interior point in $\operatorname{int}(G)$ can be removed. We would first have to trim some branches. The right picture shows a situation where no trimming is possible any more. The region is not simply connected. 

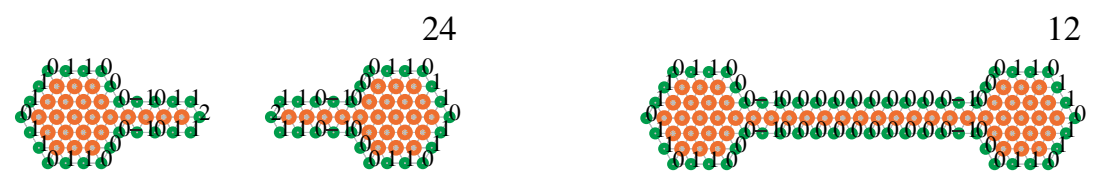

Fig. 13 Joining two regions changes the total curvature by 12 . One can prove this by trimming both sides. The left-hand side trims down to a disconnected domain consisting of two discs of radius 1 , the right-hand side will end up with a disc of radius 1.

Lemma 5. The Euler characteristic does not change under the pruning and etching operations defined above as long as the subgraphs remain smooth domains. Filling in a simply connected hole adds 1 to the Euler characteristic.

Proof. Both removing an end point of a 1-dimensional branch, as well as removing a 2dimensional point from a ridge does not change $\chi$. The number of interior points of a smooth region is $2 f-e+\chi$ as can be proven inductively: Each added face corresponds to add two edges unless we generate a new interior point, where one edge adds a face. The second statement follows from the fact that a hole $H$ in a region $G$ has $\chi(H)=1$ and that $\chi(H)+\chi(G)=\chi(H \cup G)-\chi(H \cap G)$ with $\chi(H \cap G)=0$ because $H \cap G$ is a closed path.

Theorem 6 (Discrete Gauss-Bonnet theorem). If $G$ is a finite smooth domain $G$ with boundary $C$, then

$$
\sum_{p \in C} K(p)=12 \chi(G)
$$

We could use the same pruning-etching technique as before. However, pruning and etching can lead to final situations which have no end points like a ring. Instead of classifying all these final situations, it is easier to reduce the general situation to a simply connected situation. There are two ways to change the topology: we can either build bridges between different connected components or we can fill holes to make the region simply connected. Merging different unconnected components works as long as their complements are smooth, both the Euler characteristic as well as the total curvature add up.

We can assume the region to be connected, because both the curvature as well as the Euler characteristic are additive with respect to adding disjoint domains as long as the complement is a domain. To illustrate this more, we can join two separated regions along with a 1-dimensional bridge. The curvature drops by 12 , the number of connected components drops by 1 . See Fig. 13.

Definition. A hole $W$ of $G$ is a bounded simply connected smooth region such that the interior $\operatorname{int}(W)$ is a connected component of the complement $G^{\prime}$ of $G$ and the interior $\operatorname{int}(G)$ is a subset of the complement of $W$. Holes are assumed to be simply connected since otherwise $G$ would be disconnected in which case the discussion can be delegated to each connected component of $G$. By definition, the hole $W$ and the region $G$ share a common 1-dimensional boundary. 

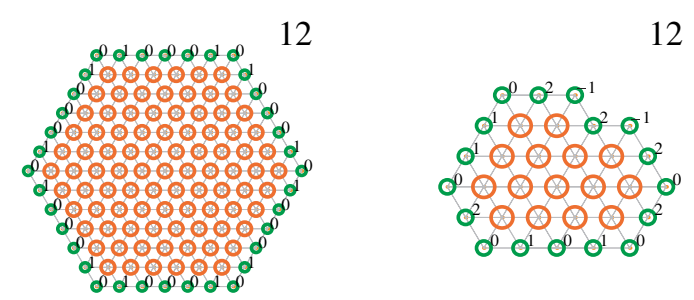

12

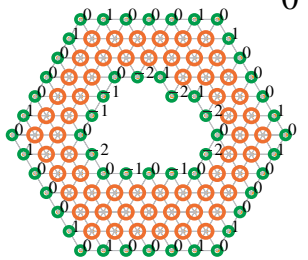

Fig. 14 Filling a simply connected hole in a larger region adds to the curvature exactly the same amount than the total curvature of the hole. The point-wise curvatures of the removed inside region matches the curvatures of the inner outside region. Filling a hole increases the curvature by 12 and the Euler characteristic by 1 .

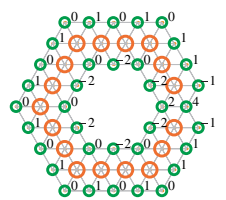

10

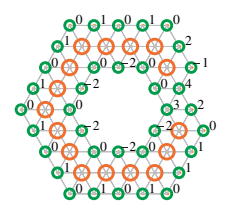

8

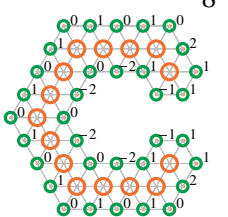

12

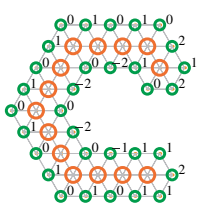

Fig. 15 When cutting a ring, the curvature changes from 0 to 12 . Only the last region is a smooth region. The second last is a region but not smooth because the complement is not a region.

By Theorem 2, the hole has total curvature 12. A key observation is that the point-wise curvatures at the inner boundary of $G$ enclosing the hole are just the negative of the corresponding point-wise curvatures of the hole. Fig. 14 illustrates this. This follows almost from the definition of curvature and the fact that the circles $\left|S_{1}(p) \cap W\right|+\left|S_{1}(p) \cap G\right|=6$ and $\left|S_{2}(p) \cap W\right|+\left|S_{2}(p) \cap G\right|=12$ so that $K_{W}(p)+K_{G}(p)=0$. Alternatively, we could also cut rings, as Fig. 15 illustrates.

\section{Compact flat graphs}

If we introduce identifications in the hexagonal background graph $X$, the topology of the background space changes. Identifying points along two parallel lines for example produces a flat cylinder. With a triangular tiling, we can tessellate a torus. Because there is no boundary now, the sum of the curvatures is zero, which is the Euler characteristic. There are many different non-isometric graphs which lead to such twisted tori. As graphs they can be different even, if the number of faces, edges, and vertices are the same.

The notion of regular domain can be carried over to subsets of such a twisted background torus $X$. We assume that it is large enough so that $S_{2}(p)$ is a circle at every point. We still have:

Theorem 7. If $G$ is a domain in a twisted torus $X$, then $\sum_{p \in \partial G} K(p)=12 \chi(G)$.

Proof. If the region is simply connected, the same proof as before applies: the domain can be trimmed down to a point. If $G$ is equal to the background torus $X$ itself, the formula holds also because there is no boundary and the Euler characteristic is 0. Removing a simply connected component of $G$ reduces the Euler characteristic by 1 . Filling a hole 

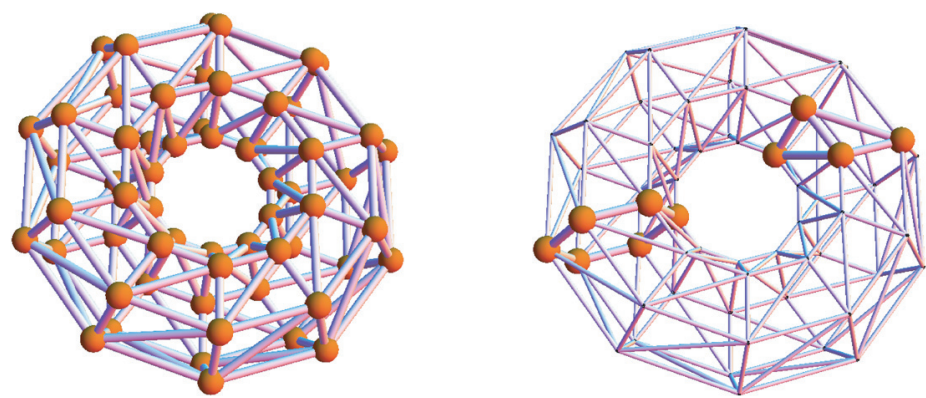

Fig. 16 The left picture shows a twisted flat torus $X$ where opposite sides of a rectangular domain in a hexagonal lattice are identified and the total curvature is 0 . On the right, the interior of a region $G$ in $X$ is shown. It has two connected components. One is simply connected with Euler characteristic 1. The closed loop has Euler characteristic 0 .

adds 1 to the Euler characteristic. After filling up all holes, all connected components of $G$ can be edged and trimmed to connected domains $G_{i}$ for which each $\operatorname{int}\left(G_{i}\right)$ is either a 1-dimensional closed graph winding around the torus or trimmed to a point. The Euler characteristic of a domain $G_{i}$ is 0 , if a trimmed down version is a closed loop or 1 , if it is a point. For every connected component $G_{i}$, the Euler characteristic is therefore the same as the sum of the boundary curvatures of $G_{i}$. Summation over all components leads to the result.

\section{Combinatorial curvature}

In this section, we consider a more elementary Gauss-Bonnet formula. The curvature is again defined by a Puiseux discretization. Since only circles of length 1 appear in the definition, it is a first order curvature.

Definition. For a 2-dimensional graph with boundary, the combinatorial Puiseux curvature is defined as $K_{1}(g)=6-\left|S_{1}(g)\right|$ for interior points and $K_{1}(g)=3-\left|S_{1}(g)\right|$ for boundary points.

For this combinatorial curvature, a proof of the Gauss-Bonnet theorem is easier. For subgraphs $G$ of the hexagonal lattice, the boundary curvature is almost trivially equal to 6 because the curvature is related to angles of the corresponding polygon: for a boundary point, $K_{1}(p) \cdot \pi / 3$ is the complementary interior angle at a vertex $p$ of the polygon. Because $\sum_{p} K_{1}(p) \pi / 3=2 \pi$ by the polygonal version of the Hopf "Umlaufsatz", we have $\sum_{p} K_{1}(p)=6 \chi(G)$.

It can be generalized for 2-dimensional graphs and more generally to 2-dimensional graphs with boundary. Since it is so closely related to the Euler characteristic, we should attribute it to Euler, even so we are not aware that Euler considered $K_{1}(g)$, nor that he looked at the dimension of a graph.

Definition. A graph $G$ is called a 1-dimensional graph without boundary, if every sphere $S_{1}(p)$ in $G$ is a 0 -dimensional graph with two points. A graph $G$ is called a 1-dimensional 
graph with boundary, if every unit sphere $S_{1}(p)$ is a 0-dimensional graph with one or two points. A point in a 2-dimensional graph is an interior point, if the unit sphere is a polygon, a 1-dimensional graph without boundary. If a 2-dimensional graph has only interior points it is called a 2-dimensional graph without boundary. A point $p$ in a 2-dimensional graph is a boundary point, if it is adjacent to an interior point and the unit sphere $S_{1}(p)$ is a connected 1-dimensional graph with boundary. A graph is called a 2-dimensional graph with boundary, if every point is either an interior point or a boundary point.

With these definitions, the boundary of a 2-dimensional graph $G$ with boundary is a 1dimensional graph without boundary. The boundary of $G$ is made of finitely many subgraphs $G_{i}=\left(V_{i}=\left\{g_{i 1}, \ldots, g_{i n_{i}}\right\}, E_{i}\right)$ with $i=1, \ldots, n$ of $G=(V, E)$ which are polygons and $n_{i}>3$. The stellated graph $H$ is obtained by adding $n$ vertices $p_{i}$ and edges $\left(p_{i}, g_{i j}\right)_{i=1, \ldots, n, j=1, \ldots, n_{i}}$. The graph $H$ is a 2 -dimensional graph without boundary.

Remark. The Euler characteristic is equal to $v-e+f+n$, where $v=|V|, e=|E|$, and $f$ is the number of triangles of the original graph $G$ and where $n$ is the difference between the number of vertices of $H$ and the number of vertices of $G$. The $n$ polygons of the original graph can be thought of as additional faces of the old graph $G$.

Theorem 8 (Combinatorial Gauss-Bonnet theorem). For every 2-dimensional graph with boundary, $\sum_{g \in G} K_{1}(g)=6 \chi(G)$.

\section{Remarks.}

a) This result does not need the rigid requirements on the domain. The graph does not have to be part of $X$.

b) The result appears in a different formulation: the Princeton Companion to Mathematics [3] mentions on page 832 the formula $\sum_{n}(6-n) f_{n}=12$ for convex polyhedra, where $f_{n}$ is the number $n$-hedral faces and the summation is over all face cardinalities which appear. This is an equivalent formulation but the Gauss-Bonnet character is less evident. The term polyhedron is often used in a much more restrictive way. In [2] the number $C(g)=d(g)-6$ is called the charge of the graph at the point $g$ and $\sum_{g \in G} C(g)=-12$ mentioned for planar graphs. For 2-dimensional graphs without boundary, we have $d(g)=\left|S_{1}(g)\right|$ and so $C(g)=-K_{1}(g)$.

Theorem 8 is graph theoretical. Most definitions of polyhedra assume an ambient Euclidean space or more structure on a graph. The book [5] discusses some of the struggles to define polyhedra. Since 2-dimensional graphs with boundary behave like polyhedra, one could more generally define a polyhedron as a graph in which a sequence of truncation and stellation operation produces a 2-dimensional graph $H$ without boundary. All Platonic solids are polyhedra in this sense and the Gauss-Bonnet theorem holds for $H$. For a cube for example, which is a 0-dimensional graph $G$ in our sense, a truncation of the eight vertices and additional stellation produces a 2-dimensional graph $H$ without boundary. The stellation has produced six new vertices of curvature -2 . The 24 other points have curvature 1 . The sum over all curvatures is $6(-2)+24=12=6 \chi(H)$. Also for a dodecahedron, a stellation produces twelve new vertices of curvature 1 , while the original vertices have curvature 0 after stellation. The octahedron and the icosahedron are 

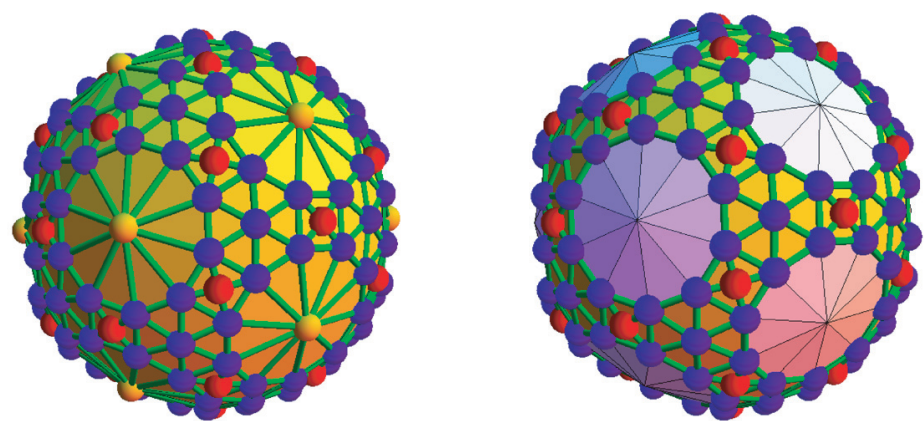

Fig. 17 The left picture shows a 2-dimensional graph $G$ with 12 points of curvature -4 and 30 points of curvature 2 leading to a total curvature of 12 . To the right, we see a graph $H$ with boundary obtained by removing all vertices of curvature -4 and their connections. Each of the 120 boundary points which are now present has curvature $-1=3-\left|S_{1}\right|$. The total curvature of $H$ is $30 \cdot 2-120=-60$ matching $6 \chi(H)$ since $\chi(H)=2-12=-10$.

already 2 -dimensional with constant curvature 2 and 1 , respectively. The tetrahedron is a 3-dimensional graph. It becomes a 2-dimensional graph $H$ without boundary after truncating its four vertices and then a stellation of the six hexagonal boundary curves. The twelve vertices have constant curvature 1, the six added face vertices have curvature 0 .

Proof. Assume first that $G=(V, E)$ is a 2-dimensional graph without boundary. Every unit sphere $S_{1}(p)$ in $G$ is then a polygon. The number of faces $f$ and the number of edges $e$ for the entire graph are then related by the dimensionality formula

$$
3 f=2 e .
$$

Additionally, for any graph without self-loops and without multiple edges, we have the edge formula

$$
\sum_{g \in V}\left|S_{1}(g)\right|=2 e
$$

The definition of the Euler characteristic combined with these two formulas gives

$$
\begin{aligned}
6 \chi(G) & =6 f-6 e+6 v=-2 e+6 v \\
& =-\sum_{g \in G}\left|S_{1}(g)\right|+6 v=-\sum_{g \in G}\left(\left|S_{1}(g)\right|-6\right)=\sum_{g \in G} K_{1}(g) .
\end{aligned}
$$

The case of a graph $G$ with boundary can be reduced to the boundary-less case: the boundary of $G$ is a union of $n$ closed cycles $G_{i}$. For each of them we add an other point $P_{i}$ and add $n_{i}=\left|G_{i}\right|$ edge connections from each of the cycle boundary points of $G_{i}$ to $P_{i}$. This produces a stellated graph $H$ without boundary and which contains $G$ as a subgraph. The formula without boundary shows that $6 \chi(H)$ is the sum of curvatures of the original interior points and the sum of the curvatures of the boundary points as well as the sum of the 
curvatures $K_{1}\left(P_{i}\right)=6-n_{i}$ to each newly added point $P_{i}$ :

$$
6 \chi(H)=\sum_{g \in \operatorname{int}(G)} K(g)+\sum_{g \in \partial G} K_{H}(g)+\sum_{i=1}^{n}\left(6-n_{i}\right) .
$$

Since we have added $n$ vertices, $|\partial G|$ edges and $|\partial G|$ faces,

$$
\chi(H)=\chi(G)+n-|\partial G|+|\partial G|=\chi(G)+n .
$$

The boundary points $g$ satisfy $K_{H}(g)=6-\left|S_{1}(g)\right|-2$ and $K_{G}(g)=3-\left|S_{1}(g)\right|$ so that $K_{H}(g)-K_{G}(g)=1$. In the stellated graph $H$ each boundary point $g$ has become an interior point for which the curvature $K_{H}(g)$ is by 1 larger than the curvature $K_{G}(g)$ as a boundary point. From the previous boundary-less case, we get

$$
\begin{aligned}
6 \chi(H) & =\sum_{g \in H} K_{H}(g) \\
& =\sum_{g \in \operatorname{int}(G)} K_{G}(g)+\sum_{i=1}^{n} \sum_{g \in G_{i}}\left(K_{G}(g)+1\right)+\sum_{i=1}^{n}\left(6-n_{i}\right) \\
& =\sum_{g \in G} K(g)+6 n .
\end{aligned}
$$

Combining this with formula (1) gives

$$
6 \chi(G)=6 \chi(H)-6 n=\sum_{g \in G} K(g)+6 n-6 n=\sum_{g \in G} K(g) .
$$

\section{About higher order curvatures}

The curvature definition $K$ is motivated by the notion of Jacobi fields [1]. While smoothness requirements are necessary for the more sophisticated second order Gauss-Bonnet formula, the metric Gauss-Bonnet formula stated in Theorem 8 holds for any polyhedron with triangular faces. For example, every finite triangularization of a 2-dimensional compact manifold works. The curvature $K=2\left|S_{1}\right|-\left|S_{2}\right|$ is more differential geometric because the Gauss-Jacobi equations $f^{\prime \prime}=-K f$ in differential geometry require the second differences of a Jacobi field $f$.

We initially were interested in extending the notion of Jacobi fields to the discrete case for numerical purposes: for a discretized Jacobi field $f$ with $f(0)=0$, we have $f^{\prime \prime}(0)=$ $f(2)-2 f(1)+f(0)=f(2)-2 f(1)$. The Jacobi equations suggest to call this $-K$. Since $f(k)$ is the variation of the geodesic when changing the angle, we can integrate over the circle and we get the arc length $\left|S_{r}\right|$ of the circle of radius $r=1$ or $r=2$. Therefore $K$ is a multiple of $2\left|S_{1}\right|-\left|S_{2}\right|$ and there is no reason to normalize this in the discrete case. The first order curvature $K_{1}=6-\left|S_{1}\right|$ on the other hand only requires first order differences. The curvature $K$ has some advantages over the curvature $K_{1}$ :

(i) The curvature formula for the boundary and in the interior is the same, while for the curvature $K_{1}$, one has to distinguish between the boundary and the interior. 
(ii) There is no reference to a flat background structure for $K$, while $K_{1}$ refers to the flat situation via integers 6 or 3 .

(iii) It is more closely rooted to the differential geometry of manifolds and to classical notions like Jacobi fields, a notion which is of second order too.

(iv) It can be adapted to higher dimension, when defining scalar curvature for graphs and where no natural flat triangulated ambient reference graph exists. While $K_{1}$ is of metric flavor, $K$ is more differential geometric.

Similarly as many metric results extend to the differential geometric setup, statements become more subtle in the discrete case, if higher order difference notions are used. The limitations of the results are related to similar limitations we know in the continuous case. We can combine the two results: for the Puiseux curvature with radius 2 defined by $K_{2}(g)=12-\left|S_{2}(g)\right|$ in the interior and $K_{2}(g)=6-\left|S_{2}(g)\right|$ at the boundary, we get the following corollary:

Corollary 9. If $G$ is a 2-dimensional smooth domain with boundary $C$ in the triangular tessellation $X$ of the plane, then $\sum_{g \in C} K_{2}(g)=24 \chi(G)$.

Proof. Add $\sum_{g \in C}\left(6-2\left|S_{1}(g)\right|\right)=12 \chi(G)$ from Theorem 8 with $\sum_{g \in C}\left(2\left|S_{1}(g)\right|-\right.$ $\left.\left|S_{2}(g)\right|\right)=12 \chi(G)$ from Theorem 6 to get $\sum_{g \in C} K_{2}(g)=\sum_{g \in C}\left(6-\left|S_{2}(g)\right|\right)=24 \chi(G)$.

For example, if $G$ is a disc of radius 1 , then $\left|S_{1}\right|=\left|S_{2}\right|=2$ for all the six boundary points $g$, where $K_{1}(g)=2$ and $K_{2}(g)=4$.

Unlike the combinatorial curvature formula $\sum_{g \in G} K_{1}(g)=6 \chi(G)$, the formula for $K_{2}$ is only obvious modulo the main result Theorem 6 for smooth domains.

\section{References}

[1] Berger, M.: A Panoramic View of Riemannian Geometry. Springer-Verlag, Berlin 2003.

[2] Bondy, J.; Murty, U.: Graph theory. Vol. 244, Graduate Texts in Mathematics. Springer, New York 2008.

[3] Gowers, T. (ed.): The Princeton Companion to Mathematics. Princeton University Press, 2008.

[4] Hopf, H.: Über die Drehung der Tangenten und Sehnen ebener Curven. Compositio Math. 2 (1935), 50-62.

[5] Lakatos, I.: Proofs and Refutations. Cambridge University Press, 1976.

Oliver Knill

Department of Mathematics

Harvard University

Cambridge, MA 02138, USA

e-mail:knill@math.harvard.edu 